\title{
Necrosis of the small intestine leading to a diagnosis of polyarteritis nodosa: a case report
}

\author{
Saori Yajima', Hiroshi Asano ${ }^{1 *}$ (D), Hiroyuki Fukano', Yasuhiro Ohara', Nozomi Shinozuka', Chih-Ping Li and \\ Taketo Yamada
}

\begin{abstract}
Background: Polyarteritis nodosa is a disease that presents with necrotizing vasculitis in small and medium-sized arteries. It may occur in various organs, but approximately half of cases have gastrointestinal involvement. Prognosis is not favorable once organ dysfunction begins as evidenced by gastrointestinal symptoms; thus, treatment with steroids should be promptly initiated. We report the case of a patient who presented with necrosis of the small intestine, which was pathologically diagnosed as polyarteritis nodosa and treated successfully with steroids.

Case presentation: An 18-year-old Japanese woman reported a sudden onset of abdominal pain and vomiting that led her to visit our emergency department, where she was evaluated by a physician. On physical examination, tenderness to palpation in the upper umbilical region was noted, and diagnostic imaging with computed tomography showed emphysema of the wall of her small intestine. She was diagnosed as having necrosis of the small intestine requiring urgent surgery. No strangulations were noted intraoperatively but approximately $20 \mathrm{~cm}$ of her small intestine was necrotized. The surrounding arteries were examined and no palpable pulse was observed; therefore, segmentectomy of the necrotized regions was performed. Pathological findings revealed active vasculitis with fibrinoid necrosis, as well as destruction, fibrogenesis, and luminal stenosis of the elastic lamina found in the muscular arteries. A diagnosis of polyarteritis nodosa was confirmed as the cause of the necrosis of her small intestine. No recurrence of polyarteritis nodosa symptoms was observed when she was administered $40 \mathrm{mg}$ of prednisolone daily.
\end{abstract}

Conclusion: In cases of idiopathic intestinal necrosis or perforation, systemic diseases such as polyarteritis nodosa should be considered in the differential diagnosis.

Keywords: Polyarteritis nodosa, Intestinal necrosis, Necrotizing vasculitis

\section{Background}

Polyarteritis nodosa (PAN) is a condition involving necrotizing vasculitis in small and medium-sized arteries [1]. It can often occur in various organs, with approximately half of patients having gastrointestinal involvement [2, 3]. Development of gastrointestinal lesions results from the formation of ulcers or erosions primarily due to ischemic changes with narrowing of the intravascular lumen. If perforation or necrosis is present, an accompanying acute abdominal condition should be considered because such

\footnotetext{
* Correspondence: hiroshia@saitama-med.ac.jp

${ }^{1}$ Department of General Surgery, Saitama Medical University, 38

Morohongou, Moroyama, Iruma-gun, Saitama 350-0495, Japan

Full list of author information is available at the end of the article
}

cases require urgent surgery. In fact, some reports state that more than half of patients with PAN with associated abdominal symptoms require surgery for an acute abdominal condition [4]. Thus, patients may first present with an acute abdominal condition that leads to a postoperative diagnosis of PAN. PAN can be treated with steroids, but if organ dysfunction occurs, as evidenced by gastrointestinal symptoms, the prognosis is poor [5] and prompt treatment is desirable. For these reasons, patients with an acute abdominal condition, such as intestinal necrosis or perforation, should be treated with the consideration that PAN may be a possibility. The present patient underwent emergency surgery for suspected strangulated intestinal obstruction, which led to a definitive diagnosis of PAN

(c) The Author(s). 2019 Open Access This article is distributed under the terms of the Creative Commons Attribution 4.0 International License (http://creativecommons.org/licenses/by/4.0/), which permits unrestricted use, distribution, and 
after surgery, but it was also possible to suspect PAN based on the preoperative findings. We report a case who presented with necrosis of the small intestine, which was pathologically diagnosed as PAN and treated successfully with steroids.

\section{Case presentation}

An 18-year-old Japanese woman experienced a sudden onset of abdominal pain and vomiting, and was therefore transported to the out-patient emergency ward at our institution. She was a student with no employment history. Her height was $156 \mathrm{~cm}$ and weight was $55 \mathrm{~kg}$. Her consciousness was normal and there were no neurological abnormalities. She had been receiving antihistamines for atopic dermatitis but had no other conditions, such as neuropathy or hematuria, which would lead us to suspect neuritis or vasculitis. She had no history of tobacco smoking or alcohol consumption. Moreover, there was nothing of note in her family history.

On physical examination, palpation revealed a flat abdomen with tenderness in the upper umbilical region, and no signs of peritoneal irritation. Her temperature was $36.3^{\circ} \mathrm{C}$, blood pressure was $159 / 123$ $\mathrm{mmHg}$, pulse was 85 beats/minute, and oxygen saturation was $99 \%$ on room air. Her blood laboratory findings revealed a white blood cell count of $8600 / \mathrm{mm}^{3}$ and a C-reactive protein level of $0.12 \mathrm{mg} / \mathrm{dL}$, which was not suggestive of an inflammatory process. However, an increased D-dimer level of $4.36 \mu \mathrm{g} / \mathrm{mL}$ was noted. Her renal and liver functions were normal (blood urea nitrogen $9 \mathrm{mg} / \mathrm{dL}$, creatinine $0.4 \mathrm{mg} / \mathrm{dL}$, aspartate aminotransferase $31 \mathrm{U} / \mathrm{L}$, and alanine aminotransferase $21 \mathrm{U} / \mathrm{L}$ ). An abdominal contrast-enhanced computed tomography (CT) scan showed emphysema of the wall of her small intestine with poor contrast enhancement that corresponded to the area of tenderness (Fig. 1). However, the contrast CT revealed heterogeneous contrast enhancement, leading to a suspicion of renal infarction (Fig. 2). Necrosis of the small intestine was suspected based on the imaging findings, and urgent surgery was performed.

Laparotomy revealed an area of necrosis $20 \mathrm{~cm}$ in length, which was approximately $100 \mathrm{~cm}$ away from the Treitz ligament of the jejunum. There were no findings of strangulation, which precluded the identification of the cause of the necrosis (Fig. 3). Since there was no palpable pulse over the arteries surrounding the necrotized intestine, the necrotized regions were resected and the small intestine reconstructed to maintain a palpable pulse.

After surgery, a thrombotic lesion was suspected and anticoagulant therapy with heparin sodium at 20,000 U/ day was started. However, pathological findings showed

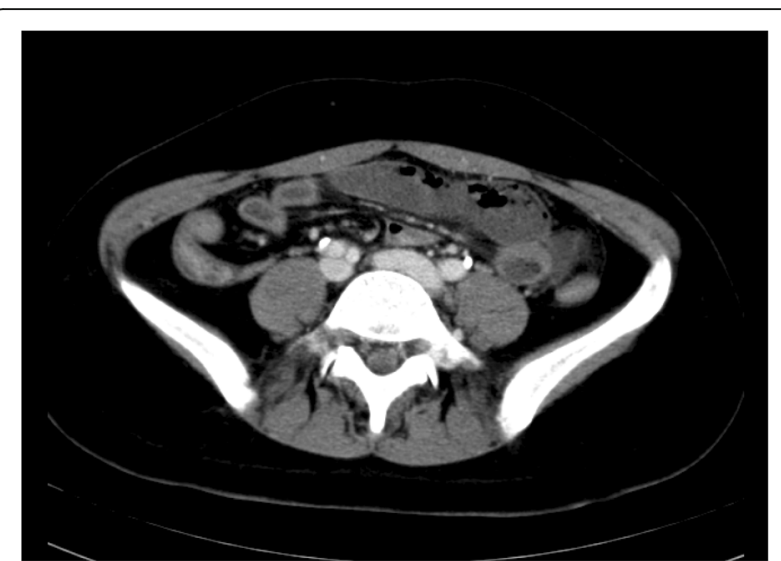

Fig. 1 Findings on abdominal contrast-enhanced computed tomography. Poor contrast enhancement of the small intestine is noted

active vasculitis associated with fibrinoid necrosis, as well as destruction, fibrogenesis, and luminal stenosis of the elastic lamina in the muscular arteries. Our patient was therefore diagnosed as having PAN-induced necrosis of the small intestine (Fig. 4). Other postoperative investigations included a blood test, which showed that she was negative for proteinase 3-antineutrophil cytoplasmic antibody (ANCA) and hepatitis B surface antigen, but weakly positive for myeloperoxidase (MPO)-ANCA $(5.3 \mathrm{U} / \mathrm{mL}$; normal range, $0.0-3.4 \mathrm{U} / \mathrm{mL})$. A postoperative angiograph of her renal arteries demonstrated multiple aneurysms in the renal arterioles. Postoperative urine analysis was positive for proteinuria. Anticoagulant therapy was discontinued, and she was administered $40 \mathrm{mg}$ of prednisolone daily starting on postoperative day 11 . Since she continued to improve, the dose of prednisolone was reduced. It has been a year since the surgery, and she is receiving prednisolone at $5 \mathrm{mg}$ a day without recurrence.

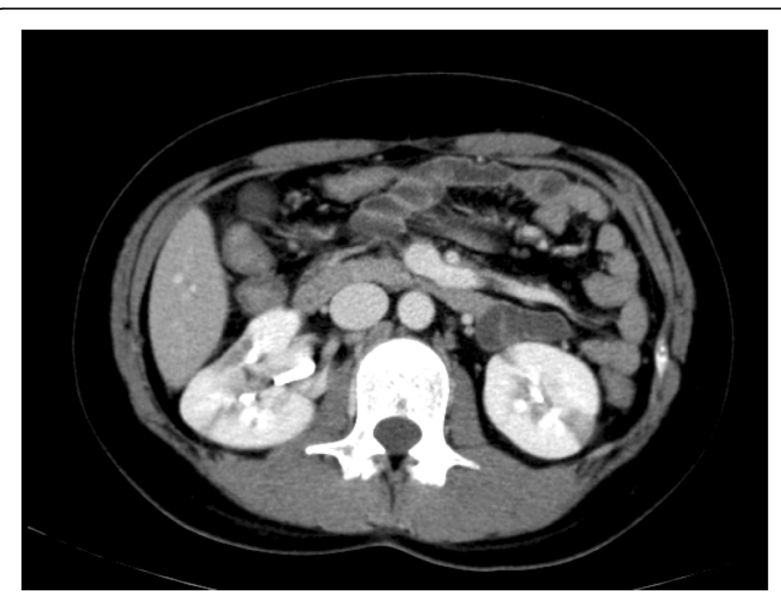

Fig. 2 Findings on abdominal contrast computed tomography. Regions with poor contrast enhancement are noted in both kidneys 


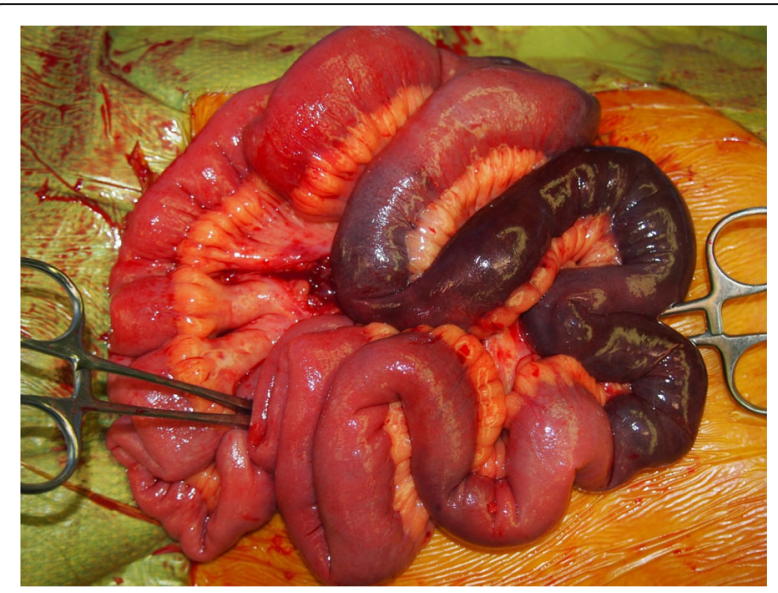

Fig. 3 Intraoperative findings. The small intestine with approximately $20 \mathrm{~cm}$ of necrotized tissue
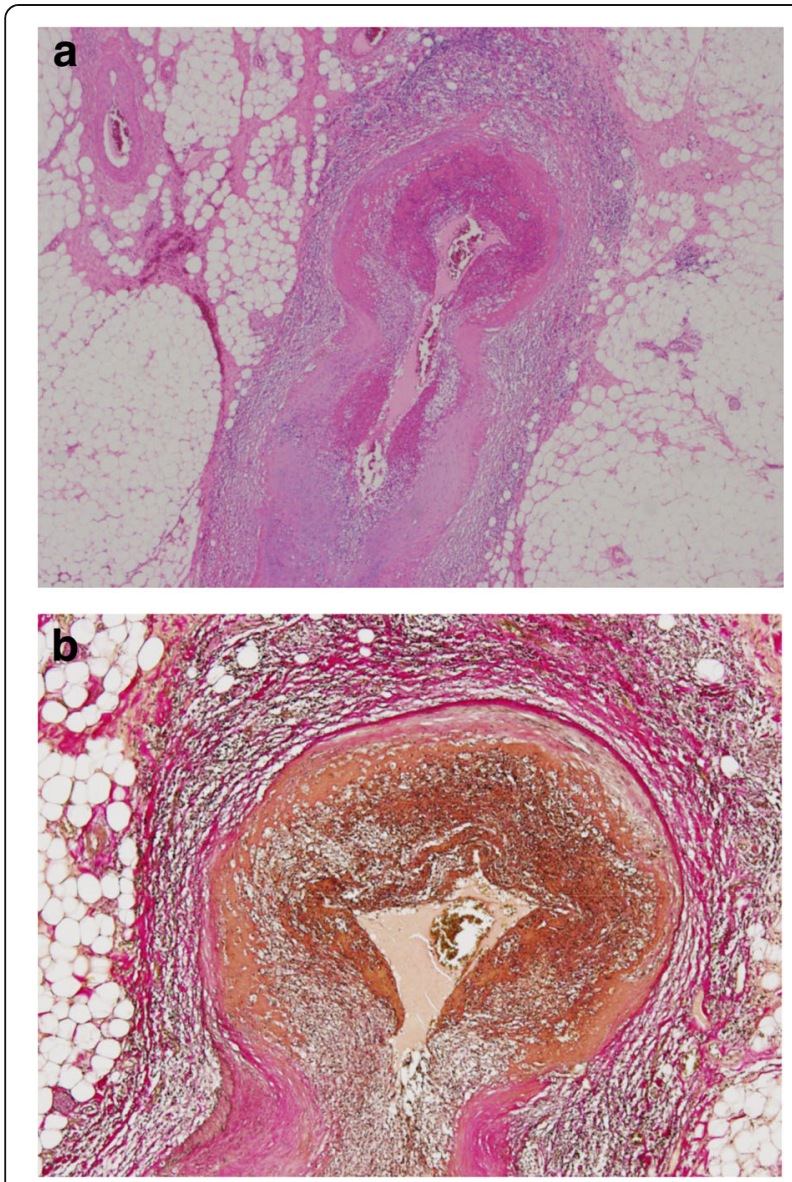

Fig. 4 Histopathological findings. a Active vasculitis associated with fibrinoid necrosis was seen in the mesenteric artery $(\times 20)$. b Elastica van Gieson staining showed destruction, fibrogenesis, and luminal stenosis of the elastic lamina $(\times 40)$

\section{Discussion}

In this case, emergency surgery was performed based on a diagnosis of necrosis of the small intestine, caused by an internal hernia or strangulated intestinal obstruction, and PAN was diagnosed pathologically after the operation. Although PAN is often accompanied by gastrointestinal symptoms, few cases develop due to intestinal necrosis. Similar to this case, emergency surgery based on a diagnosis of non-occlusive mesenteric ischemia [6] or mesenteric artery thrombosis [7] has been reported. Previous studies also indicate that diagnosing PAN preoperatively in the presence of an acute abdomen is difficult and that it is commonly diagnosed after postoperative pathological examination. While preoperative diagnosis was not possible in this case, in retrospect, PAN may have been suspected preoperatively based on the presence of renal infarction. As early steroid therapy improves the prognosis of PAN, early consideration should be given to systemic diseases when diagnosing cases of intestinal necrosis.

PAN is a systemic vasculitis disorder that presents with necrotizing vasculitis in small and medium-sized arteries, without arteriolitis or capillaritis. Patients with PAN are also classically MPO-ANCA-negative. However, MPO-ANCA-positive cases involve vasculitis of the arterioles and capillaries, and are therefore diagnosed as microscopic polyangiitis. However, there have been reports of MPO-ANCA-positive PAN cases [8, 9]. These cases were diagnosed as PAN because the pathological findings showed necrotizing vasculitis in small and medium-sized arteries despite being MPO-ANCA-positive. In fact, in the present case, angiographic and pathological findings indicated lesions in the arterioles; thus, our patient was diagnosed as having PAN despite MPO-ANCA positivity.

Patients with PAN show a wide variety of symptoms, such as fever, weight loss, malaise, multiple arthralgia, myalgia, and muscle weakness. PAN can be diagnosed based on these clinical findings, as well as by the presence of necrotizing arteritis in the muscular arteries on histological investigation. It often occurs in various organs, with the kidneys more likely to be affected. This results in the onset of renal failure due to vasculitis-induced renal infarction. The digestive organs are also common sites of PAN, with half of patients with the disease having gastrointestinal lesions. Lesions of the small intestine are frequently present, but lesions can also occur in the large intestine, liver, pancreas, and gallbladder [8].

Gastrointestinal lesions are characterized by narrowing of the intravascular lumen due to vasculitis, with the formation of ulcers or erosions observed on gross pathology. Patients with PAN commonly suffer from non-specific symptoms, such as abdominal pain, nausea, or diarrhea, 
with some reports showing that $14-25 \%$ of patients with PAN-related gastrointestinal lesions initially experience gastrointestinal symptoms $[9,10]$. As the lesions progress, bleeding or perforation can lead to ischemia and, eventually, necrosis. Levine et al. [4] investigated 24 cases of PAN with gastrointestinal symptoms and found that $54 \%$ had acute abdominal conditions requiring surgery, including eight that had progressed to necrosis or perforation and four with bleeding caused by a ruptured aneurysm.

According to Bourgarit et al. [5], there are five risk factors that impact the prognosis of PAN, including uric protein $>1 \mathrm{~g} /$ day, serum creatinine levels $>1.58 \mathrm{mg}$, prior gastrointestinal surgery, cardiomyopathy, and a central nervous system disorder. In Bourgarit et al.'s study, the 5-year survival rate was $88 \%$ in patients with zero risk factors, which decreased to $55 \%$ in those with three or more risk factors. Pagnoux et al. [3] reported on 62 cases with necrotizing vasculitis, including 38 cases of PAN with a 5 -year survival rate of $76 \%$, among which $50 \%$ of mortalities were caused by gastrointestinal lesions. Other reports have revealed a 5-year survival rate of $67 \%$ in cases without gastrointestinal lesions, and $55 \%$ in those complicated with gastrointestinal lesions [11]. As mentioned above, the prognosis of PAN is not encouraging; thus, careful follow-up including recurrence prevention is required, particularly for cases complicated by gastrointestinal lesions.

Regarding treatment, the steroids prednisone or prednisolone are used, with doses usually starting at $1 \mathrm{mg} / \mathrm{kg}$ per day. The dose can then be reduced as needed if the patient's condition improves. However, in cases where disease recurrence precludes steroid reduction or where organ dysfunction has occurred, the combined use of immunosuppressive agents may be considered. In addition, in cases of life-threatening conditions or rapid disease progression, steroid pulse therapy may be applied [2]. In this case, our patient was started on $40 \mathrm{mg}$ of prednisolone on postoperative day 11 and she has had no recurrence of gastrointestinal lesions in the remaining intestinal regions. Since her condition was stable, no immunosuppressive agents were administered.

\section{Conclusion}

Since PAN is associated with a high incidence of gastrointestinal lesions, an acute abdominal condition may occur as the initial presentation. A pathological assessment is required for the diagnosis of PAN. In cases of intestinal necrosis or perforation where intraoperative findings cannot clarify the cause, sample collection is necessary to definitively diagnose a systemic disease such as PAN.

\section{Abbreviations}

ANCA: Antineutrophil cytoplasmic antibody; CT: Computed tomography: MPO-ANCA: Myeloperoxidase-antineutrophil cytoplasmic antibody; PAN: Polyarteritis nodosa

\author{
Acknowledgements \\ None. \\ Funding \\ None. \\ Availability of data and materials \\ Data sharing is not applicable to this article, because no datasets were \\ generated or analyzed during the present study.
}

\section{Authors' contributions}

SY and HA performed the operation. HF, YO, and NS participated in the treatment of this patient and revised the manuscript. $\mathrm{CL}$ and TY performed the pathological diagnosis. All authors read and approved the final version of this manuscript

\section{Ethics approval and consent to participate}

Not applicable.

\section{Consent for publication}

Written informed consent was obtained from the patient for publication of this case report and any accompanying images. A copy of the written consent is available for review by the Editor-in-Chief of this journal.

\section{Competing interests}

The authors declare that they have no competing interests.

\section{Publisher's Note}

Springer Nature remains neutral with regard to jurisdictional claims in published maps and institutional affiliations.

\section{Author details}

${ }^{1}$ Department of General Surgery, Saitama Medical University, 38 Morohongou, Moroyama, Iruma-gun, Saitama 350-0495, Japan. ${ }^{2}$ Department of Pathology, Saitama Medical University, 38 Morohongou, Moroyama, Iruma-gun, Saitama 350-0495, Japan.

Received: 23 July 2018 Accepted: 17 February 2019

Published online: 09 March 2019

\section{References}

1. Jennette JC. Overview of the 2012 revised International Chapel Hill Consensus Conference nomenclature of vasculitides. Clin Exp Nephrol. 2013;17:603-6.

2. Hernández-Rodríguez J, Alba MA, Prieto-González S, Cid MC, HernandezRodriguez J, Alba MA, et al. Diagnosis and classification of polyarteritis nodosa. J Autoimmun. 2014;48-49:84-9.

3. Pagnoux C, Mahr A, Cohen P, Guillevin L. Presentation and outcome of gastrointestinal involvement in systemic necrotizing vasculitides. Medicine. 2005;84:115-28.

4. Levine SM, Hellmann DB, Stone JH. Gastrointestinal involvement in polyarteritis nodosa (1986-2000): Presentation and outcomes in 24 patients. Am J Med. 2002;112:386-91.

5. Bourgarit A, Le Toumelin P, Pagnoux C, Cohen P, Mahr A, Le Guern V, et al. Deaths occurring during the first year after treatment onset for polyarteritis nodosa, microscopic polyangiitis, and Churg-Strauss syndrome: a retrospective analysis of causes and factors predictive of mortality based on 595 patients. Medicine. 2005;84:323-30.

6. Shirai T, Fujii H, Saito S, Ishii T, Yamaya H, Miyagi S, et al. Polyarteritis nodosa clinically mimicking nonocclusive mesenteric ischemia. World J Gastroenterol. 2013;19:3693-8.

7. Hiraike $Y$, Kodaira M, Sano M, Terazawa Y, Yamagata S, Terada S, et al. Polyarteritis nodosa diagnosed by surgically resected jejunal necrosis following acute abdomen. World J Gastroenterol. 2013;19:2830-4. 
8. Takada T, Yoshida H, Tsukuda M, Katoh H. A case of classic polyarteritis nodosa presenting as acute abdomen. A review of 50 patients with PN involving small intestine undergone laparotomy in Japanese literature. Jpn J Gastroenterol Surg. 2003;36:51-6.

9. Agard C, Mouthon L, Mahr A, Guillevin L. Microscopic polyangiitis and polyarteritis nodosa: How and when do they start? Arthritis Rheum. 2003;49:709-15.

10. Frohnert PP, Sheps SG. Long-term follow-up study of periarteritis nodosa. Am J Med. 1967;43:8-14.

11. Guillevin L, Du LTH, Godeau P, Jais P, Wechsler B. Clinical findings and prognosis of polyarteritis nodosa and Churg-Strauss angiitis: a study in 165 patients. Br J Rheumatol. 1988;27:258-64.

Ready to submit your research? Choose BMC and benefit from:

- fast, convenient online submission

- thorough peer review by experienced researchers in your field

- rapid publication on acceptance

- support for research data, including large and complex data types

- gold Open Access which fosters wider collaboration and increased citations

- maximum visibility for your research: over $100 \mathrm{M}$ website views per year

At BMC, research is always in progress.

Learn more biomedcentral.com/submissions 